being a tributary of the former, and both draining ultimately into the Yukon. The coals are of JurassoCretaceous age. The physiography and natural history of the district are described, and the summer months are said to be delightful. The drift-filled valley floors, set with little lakes, are likely to attract stock-raisers and even cultivators.

Messrs. F. D. Adams and A. E. Barlow furnish an important memoir of some 400 pages (No. 6, price 30 cents) on the Haliburton and Bancroft areas of Ontario. This region is part of a great elevated plain, dissected into moderate and rolling hills, and formed of Laurentian granite-gneisses, with inclusions of amphibolite. The authors give conclusive reasons for regarding the latter masses as derived from the roof or walls of the granite batholith; the blocks fell into the magma, and partook of its subsequent movements (p. $12 \mathrm{I}$ ). Frequently they become streaked out, until strongly banded and even contorted gneisses are produced (Fig. 2); but the authors hesitate (p. 123) to urge that all the grey gneiss of the district has originated in this composite way. The amphibolites, even on a large scale, are traced to the contactalteration of limestones by the granite, with perhaps some intermingling from the igneous magma. This is, of course, what has been urged in many other areas; but the broad exposures in these undulating Canadian lands offer unusual opportunities for demonstration. Many other types of crystalline rock are described, including syenites with corundum, and the whole memoir is one of immense interest to the petrographer. The corundum is worked commercially (p. $37 \mathrm{r})$.

Mr. J. Dresser describes St. Bruno Mountain, in Quebec, in Memoir 7, a mass of ultrabasic igneous rock intruded into Ordovician rocks, probably in Devonian times. Mr. D. Dowling deals, in Memoir 8-E, with the promising field of Cretaceous and Cainozoic coals at Edmonton, on the Saskatchewan, in the north-west.

Memoir 3 is a quarto by Mr. L. M. Lambe on Palæoniscid fishes from the Albert Shales of New Brunswick. The author correlates these beds (p. 14) with the Scotch Calciferous Sandstone. He figures, among other specimens, the types that were described by C. T. Jackson, without illustrations, in $185 \mathrm{r}$.

Mr. C. D. Walcott (Smithsonian Miscell. Collections, vol. liii., No. 7,1910 ) has carried his studies of Cambrian stratigraphy into the Bow River Valley, Alberta, Canada, a highly picturesque and mountainous region, where he finds that the basal Cambrian beds rest unconformably on unaltered pre-Cambrian shales and sandstones.

Perhaps we may mention here, in conclusion, a paper by Mr. R. Guppy, published in Canada (Trans. Canadian Institute, vol. viii., p. 373), on "The Geological Connections of the Caribean Region." Mr. Guppy, writing from Trinidad, discusses deep-water Cainozoic beds in Jamaica and other islands, and argues for a former "land connection between the Caribean and North Africa and a sea connection between the Caribean Sea and the Pacific." Both these probably passed away at the close of Miocene times. Mr. Guppy has a way of abolishing double letters in generic names, which may be American, but is hardly fair to their originators.

G. A. J. C.

\section{PRESSURE IN STELLAR ATMOSPHERES}

$W$ ITHIN the last fifteen years the spectroscopic equipment applied to the study of both laboratory and celestial investigation has been very materially modified both in dispersive power and design. In the early 'nineties there were very few of the $2 \mathrm{I} \cdot 5$-feet Rowland concave grating spectrographs in regular commission for terrestrial research, and it is probably safe to say that no stellar spectrographs were in use giving direct spectra comparable in dispersion with Rowland's solar spectrum.

The transference of Dr. G. E. Hale's sphere of labours from the Yerkes Observatory to the new solar observatory on the summit of Mount Wilson, Pasadena, California, in 1905, however, marks an important epoch in the progress of spectroscopy, as from that time may be dated the successful application of high dispersive spectrography to the problems of celestial and terrestrial identification, both No. 2 I67, VOL. 86] chemical and physical. Many, of the beautiful discoveries have already been noted as they were announced: the photography of sun-spot spectra on a sufficiently large scale to serve as a standard map for future reference, the magnetic field accompanying the sun-spot vortices, the detailed laws of solar rotation, all giving an enormous mass of detailed evidence from which fruitful discussions may be profitably initiated. In the examination of certain of the solar photographs numerous peculiarities were noticed in the relative wave-lengths of the lines, indicating the operation of a definite law. Some time previously Halm had announced (Astronomische Nachrichten, Bd. 173, p. 273, 1907) that certain iron lines were relatively displaced to the red at the sun's limb compared with their position at the sun's centre. In Igio Hale and Adams described the results of a long series of determinations of these minute displacements made with the spectrograph attached to the tower telescope of the solar observatory (Astrophys. Journ., vol. xxxi., p. 30, r910). After eliminating all the known differences owing to rotation and orbital motion, they found from an examination of 470 lines between $\lambda 3741$ and $\lambda 6573$ that the residual displacements could be classified to a certain extent. Thus the hydrogen lines, calcium $(\mathrm{H}, \mathrm{K}, 4227)$, sodium $\mathrm{D}$, and magnesium $b$ lines showed no appreciable displacements, and this was also the case with the lines the intensities of which were greatly strengthened at the limb of the sun. The lines of titanium, vanadium, and scandium show considerably smaller displacements than the lines of iron and nickel.

The elements of high atomic weight, such as lanthanum, cerium, in general exhibited very small displacements.

The enhanced lines, as a class, showed decidedly larger shifts than the corresponding arc lines.

All these facts point to the suggestion that they are caused by the varying pressure in different parts of the sun's atmosphere, and it will thus be readily seen how, by a careful study of these interrelations and the laboratory variations known to exist under different modes of treatment, we may find it possible to arrive at a satisfactory explanation of the mechanism of the solar atmosphere.

Now to the astrophysicist the sun is simply our nearest star, presenting to us special facilities for local selective examination by reason of the fact of its having a disc of appreciable diameter, and furnishing abundance of light. When the problem is extended to the case of stellar atmospheres the difficulties are at once greatly increased.

It was to afford the means of attacking this question in an efficient manner that Dr. Hale planned the installation of a very high dispersion spectrograph to be used with the new reflecting telescope of 60 inches aperture which has been so perfectly designed, constructed, and adjusted into working trim by Prof. G. W. Ritchey. With the new spectrograph, the spectra of several of the brighter stars have been successfully photographed, and from a preliminary study of those of $\alpha$ Canis Majoris (Sirius), $\alpha$ Canis Minoris (Procyon), and $\alpha$ Böotis (Arcturus), Mr, W. S. Adams has been enabled to come to some interesting conclusions respecting the conditions existing in the atmospheres of these stars (Astrophys. Journ., vol. xxxiii., p. 64, IgIr; Contributions from the Mount Wilson Solar Observatory No. 50 ).

By means of subsidiary mirrors, the equatorial reflector is employed in the coudé form, the light being reflected down and through the hollow polar axis to the slit of the spectrograph. The equivalent focal length of the combination of mirrors is 150 feet $(45.7$ metres), giving an aperture ratio of $1: 30$.

For convenience of manipulation and constancy of temperature, the spectrograph was arranged vertically downwards in an underground pit. The spectrograph is of the Littrow or auto-collimation type, consisting of a lens of $15.2 \mathrm{~cm}$. aperture and 5.5 metres focal length, used in conjunction with a dense flint-glass prism of $63^{\circ}$ angle, and a plane mirror to send the light back through the prism, thus giving the equivalent dispersion of two prisms. The large scale of the dispersion thus provided will be evident from the approximate linear equivalents, given as follows :

$$
\begin{aligned}
& \text { At } \lambda 4300, \mathrm{I} \mathrm{mm} \text { on photographic plate }=\mathrm{I}_{4} \mathrm{~A} \\
& \text {, 5000, ,, ,, , , },=2.4 \AA \text {. } \\
& \text {,6500, ,, , , , , }=6.2 \mathrm{~A} \text {. }
\end{aligned}
$$


Satisfactory definition is obtained throughout the whole length $(43 \mathrm{~cm}$.) of the plate used in the spectrograph camera. In the more refrangible portions of the spectrum Lumière "Sigma" plates were employed, while from $\lambda 4900$ to the red end Seed "Gilt Edge 27" plates sensitised by Wallace's formula were used. For comparison the spectrum of the iron arc was photographed alongside the star spectrum.

Six plates of the spectrum of Sirius $(\lambda 4200$ to $\lambda 6600$ ), four of the spectrum of Procyon ( $\lambda 4200$ to $\lambda 4900$ ), and nine plates of the spectrum of Arcturus ( $\lambda 4300$ to $\lambda$ 6600) were available for measurement, and the chief object in the study of these spectra has been to test the possibility of detecting any differences of displacement for the different lines, and thereby obtain some idea of the effective pressure in the atmospheres of the stars. In Sirius the number of lines available for measurement was comparatively small; in the case of Procyon and Arcturus the selection of lines was similar to those used in the investigation of similar displacements of the lines in the spectrum of the sun's limb.

The enhanced metallic lines, it will be remembered, show as a class most definitely larger shifts at the sun's limb than the ordinary arc lines. Now in the spectrum of Sirius the enhanced lines form a prominent feature, while the arc lines are few. In Procyon the enhanced lines are less prominent, while the arc lines have become more pronounced. In Arcturus the enhanced lines are almost evanescent, while the arc lines, which are associated with the spectrum of sun-spots, are very strongly developed. $\mathrm{Mr}$. Adams gives a table showing in summary form the main results of the inquiry, from which it is seen that in all cases the enhanced lines show a decided displacement to the red relative to the arc lines. Giving the displacements as radial velocities in kilometres, we may summarise the results as :-

$$
\begin{aligned}
& \text { Sirius: Enhanced-Arc Lines }=+0.90 \mathrm{~km} .=+0.014 \mathrm{~A} \\
& \text { Procyon: ", ", , }=+0.58,=+0.009 \\
& \text { Ariturus: ", ", }=+0.08,=+0.001
\end{aligned}
$$

The behaviour of the prominent lines in Arcturus is so definite that a special discussion is given of them. A large proportion of the lines of titanium, vanadium, and calcium are greatly strengthened, the enhanced lines decidedly weakened, and those of iron and chromium either strengthened or weakened according to their temperature gradation. The lines of nickel appear to be more prominent in the star spectrum than in sun-spots. The following table summarises this discussion :-

$$
\begin{array}{ccccccc}
\text { Element } & & & \begin{array}{c}
\text { Displacement } \\
\AA
\end{array} & & & \begin{array}{c}
\text { Equivalent } \\
\text { Velocity } \\
\text { km. }
\end{array} \\
\mathrm{H} & \ldots & \ldots & -0.020 & \ldots & \ldots & -\mathbf{1} 2 \\
\mathrm{Ca} & \ldots & \ldots & -0.017 & \ldots & \ldots & -0.70 \\
\mathrm{Mg} & \ldots & \ldots & -0.01 \mathrm{I} & \ldots & \ldots & -0.68 \\
\mathrm{~V} & \ldots & \ldots & -0.006 & \ldots & \ldots & -0.24 \\
\mathrm{Ti} & \ldots & \ldots & -0.006 & \ldots & \ldots & -0.23 \\
\mathrm{Ni} & \ldots & \ldots & -0.006 & \ldots & \ldots & -0.22 \\
\mathrm{Fe} & \ldots & \ldots & +0.006 & \ldots & \ldots & +0.25
\end{array}
$$

The shifts evidently suggest definite grouping of similar elements. The iron lines show a shift towards the red compared with all the other elements examined.

Such is the material Mr. Adams provides for his investigations. In the absence of any other known probable cause, he considers pressure as the principal agent causing these systematic displacements in stellar spectra. The laboratory experimental results of Humphreys and others gave as an average shift for the arc lines of iron $0.0025 \AA$ per atmosphere of pressure. At the sun's limb the enhanced lines in the more refrangible portion of the spectrum were found to be shifted approximately 50 per cent. more than the arc lines, and recent work by Mr. Gale on the spectrum of titanium indicates that the enhanced lines of this substance are also shifted more than the arc lines at the same pressure. Assuming, then, that a similar relationship exists between the enhanced and arc lines of other elements, this affords a means of estimating the gravitational pressures in the atmospheres of stars the spectra of which show these displacements. Thus, as seen in the table quoted above, the enhanced lines in the spec- trum of Sirius are shifted towards the red relative to the arc lines by $0.014 \AA$. This would correspond to a pressure of 12 atmospheres in excess of that existing in the sun's reversing layer. Similar reasoning in the case of Procyon indicates a pressure of 7 atmospheres over that of the sun's reversing layer. These results appear to be in accord with the modern view of regarding stars of the Sirian type as possessing no true photosphere, being simply a mass of gas increasing in density towards the centre without any surface of discontinuity or condensation. In such a star the light coming from great depths would most probably be visible from outside, and indications of great pressure would then be expected. In Procyon the spectrum indicates a transition stage between Sirius and the sun, and the pressure is shown intermediate also. It should be noted here, however, that one of the most important cases investigated by Humphreys in his work on pressure effects is directly opposed to the above conclusions. $\mathrm{He}$ found that in the case of calcium the blue $g$ line was shifted by pressure about twice as much as the $\mathrm{H}$ and $\mathrm{K}$ violet lines. Now the behaviour of these lines in the laboratory, and also in the spectrum of the solar chromosphere, indicates that $\mathrm{H}$ and $\mathrm{K}$ are typical enhanced lines, while $4226(g)$ is a very typical arc line. The differential pressure effect on the enhanced and arc lines of strontium was exactly similar to that of calcium, viz. the enhanced lines were shifted less than the arc lines. Unfortunately for this discussion, Humphreys only employed the arc spectrum in his pressure investigation, so that the general behaviour of the enhanced lines of other substances than calcium and strontium cannot be inferred from his results.

Passing on to the conditions of pressure in Arcturus, it is pointed out that the facts indicate the existence of a well-formed photosphere, the light from which proceeds from relatively low-pressure areas at moderate depths. The results for the lines of different elements indicated in the table are similar to those found for the solar lines. Thus in the sun hydrogen rises to very great heights, calcium and magnesium also being high-level substances Titanium is also relatively high-level, but iron is distinctly a low-level element. In Arcturus the displacements indicate exactly such an arrangement, and it is thus concluded that the lines of $\mathrm{H}, \mathrm{Ca}, \mathrm{Mg}, \mathrm{Ti}, \& \mathrm{c}$., are subject to less pressure than those of iron, and therefore that the gases producing them lie at a higher average level.

Charles P. Butler.

\section{RECENT INVESTIGATIONS ON SOIL FERTILITY.}

FOR some years past the United States Department of Agriculture Bureau of Soils has maintained that infertility might, and not unfrequently does, arise from the presence in the soil of toxic organic substances that have been excreted from the roots of plants. This view has been opposed on two grounds: it is not evident that plants do normally excrete poisonous substances; and if such substances are present there is no proof that they would act as poisons in the soil, which possesses a remarkable power of withdrawing dissolved substances from solution. Not long ago Scheiner isolated dihydroxystearic acid from a considerable number of unproductive soils, and now, in conjunction with J. J. Skinner, ${ }^{1}$ he has examined its behaviour to plants in water cultures. In all cases its effect was toxic, but the toxicity was much reduced when fertilisers were added to the solution, and was at a minimum when the fertilising constituents were present in the ratio most favourable to plant growth. Several incidental questions were also cleared up dealing with water cultures-perhaps the most difficult of all experiments to interpret-and the paper contains a great number of data bearing on the subject. The behaviour of this acid in the soil is not touched upon, and very wisely no attempt is made to argue from a water culture to a soil. It is, however, a distinct step in advance that an acid has been isolated from certain soils and identified, and shown to be poisonous in water culture. The results may well be connected with the known fact that, in absence of lime, soil becomes acid and loses fertility, which can only be restored by addition of lime or chalk.

1 Bulletin 70 , Bureau of Soils, U.S. Department of Agriculture.

NO. $2 \times 67$, VOL. 86$]$ 